\title{
Evaluación de la sedimentación del reservorio San Lorenzo y su comportamiento en la capacidad de almacenamiento cumplidos los 50 años de vida útil
}

\author{
Evaluation of sedimentation in the San Lorenzo reservoir and its behavior in the storage capacity \\ once reached the 50 years of useful life
}

Teresa Velásquez B. ${ }^{1}$; Carlos Contreras F. ${ }^{2}$

\section{Resumen}

El reservorio San Lorenzo es una estructura de almacenamiento de agua ubicada sobre el lecho del río Chipillico, en el distrito Las Lomas, de la provincia y departamento de Piura, aproximadamente a $115 \mathrm{~km}$ Noreste de la ciudad de Piura. Inició sus operaciones en el año 1959 para atender la demanda agrícola de la jurisdicción de la irrigación y colonización San Lorenzo, que era de aproximadamente 57.498,10 ha, es decir, parte de los valles Chipillico (Las Lomas) y Medio Piura (Tambogrande y Piura). El reservorio San Lorenzo almacena un volumen bruto de 258,0 MMC, con una capacidad neta de 252,7 MMC y una superficie de espejo de agua de $16 \mathrm{~m}^{2}$, el volumen muerto inicial fue de 3,0 MMC. Actualmente, el reservorio San Lorenzo tiene una sedimentación que representa el 20,4 \% de su volumen útil, es decir 51,52 MMC. La presente investigación realizó la batimetría del reservorio San Lorenzo (2008), cuyos resultados fueron que este reservorio tiene una profundidad máxima de 38,00 m, un volumen de regulación de 201,17 MMC y una superficie de espejo de agua de 15.459.029,12 $\mathrm{m}^{2}$. Se usó el método de Graf para la determinación del transporte total de sedimentos de los ríos Quiroz y Chapillico, hacia el embalse San Lorenzo. Los resultados indican que el volumen de sedimentos transportado por el río Quiroz es de 4,263 MMC y de 4,905 MMC para el río Chipillico, mientras que el Canal Quiroz transporta un volumen de sedimento equivalente a 2,021 MMC. El método de Graf menciona que los volúmenes obtenidos no son los que el río transporta, sino es la capacidad máxima que pueda transportar el río, teniendo la disponibilidad de sedimentos todo el tiempo. Es por esto que los valores obtenidos por el método de Graf fueron enmendados a la luz de un factor de corrección, obtenidos por la división entre los valores del volumen total de la batimetría del último año (2008) y los valores de los volúmenes transportados por el río Chipillico y el canal Quiroz. La reconstrucción y el rediseño del desarenador del canal Quiroz ayudarán a reducir el ingreso de sedimentos al reservorio San Lorenzo en un 35 a $40 \%$.

Palabras clave: reservorio; almacenamiento; sedimentos; batimetría.

\begin{abstract}
San Lorenzo reservoir is a water storage structure located on the riverbed in the district Chipillico- Las Lomas, Piura department, approximately $115 \mathrm{~km}$ North - East of the city of Piura. In 1959 began its operation and water agricultural demand within the jurisdiction of the Irrigation and Colonization San Lorenzo with about 57.498,10 ha, part of the valleys Chipillico (Las Lomas) and Middle Piura (Piura and Tambogrande). The San Lorenzo reservoir stores a gross volume of 258,0 MMC with a net capacity of 252,7 MMC and a surface of $16 \mathrm{~m}^{2}$. The initial void volume was 3,0 MMC. Currently the reservoir San Lorenzo has a sedimentation representing 20,4\% of its working volume, which means 51,52 MMC. This research performed the bathymetry of the San Lorenzo reservoir, the results showed that this reservoir has a maximum depth of $38,00 \mathrm{~m}$, a regulation volume of 201,17 MMC and a water surface of $15.459 .029,12 \mathrm{~m}^{2}$. We used the Graf method for the determination of total sediment transportation of the Quiroz and Chapillico rivers, to the San Lorenzo reservoir. The results indicate that the volume of sediment transported by the Quiroz River is 4,263 MMC and 4,905 MMC for the Chipillico River, while the Quiroz Channel carries a sediment volume equivalent to 2,021 MMC. The method of Graf mentions that the volumes obtained are not the volumes that the river carries, these represent the maximum capacity that the river can transport, having sediment availability all the time. This is why the values obtained by the method of Graf were corrected by a correction factor, obtained by the division between the total volume values from the bathymetry of the last year (2008) with the values of the volumes transported by the Chipillico River and the Quiroz channel. The reconstruction and redesign of the Quiroz channel desander will help to the reduction of sediment entering the San Lorenzo reservoir by 35 to $40 \%$.
\end{abstract}

Keywords: reservoir; storage; sediment; bathymetry.

1 Docente del Departamento de Recursos Hídricos - FIA. Universidad Nacional Agraria La Molina, Lima, Perú. Email: tvelasquez@lamolina.edu.pe 2 Tesis para optar el Título de Ingeniero Agrícola. Universidad Nacional Agraria La Molina, Lima, Perú. 


\section{Introducción}

En las últimas tres décadas y como consecuencia de la presencia del Fenómeno de El Niño, se ha observado con bastante preocupación como los principales embalses de almacenamiento de agua del país (Poechos, San Lorenzo, Tinajones y Gallito Ciego), experimentaban un proceso acelerado de sedimentación y pérdida de la capacidad de almacenamiento.

La sedimentación de reservorios es un fenómeno geomorfológico mediante el cual los sólidos transportados por las corrientes de los ríos son retenidos, depositados y compactados en el vaso. En general, cuando se va a realizar un estudio de sedimentos, no se cuenta con la información necesaria sobre su transporte en los cursos de agua y en el sitio de interés del estudio, lo cual dificulta la factibilidad operativa del proyecto hidráulico que depende, fundamentalmente, de la apreciación correcta del trasporte de sedimentos al embalse (Lezcano-Rocha, 1969).

Para la evaluación adecuada de los efectos del transporte de sedimentos, se requiere contar con estimativos suficientemente precisos del tipo, magnitud y variación del transporte de sólidos por las corrientes de agua que llegan al embalse. Las formas de cuantificar los volúmenes colmatados son realizadas de diversas maneras, entre las más empleadas tenemos: la batimetría del embalse y el muestreo del transporte sólido del río.

La sedimentación del reservorio San Lorenzo no es ajeno a los problemas de pérdida de su capacidad de almacenamiento; el proceso de erosión hídrica y sedimentación está relacionado con el intemperismo, altas pendientes de los taludes de las quebradas, altas precipitaciones, prácticas agrícolas inadecuadas, deforestación, sobrepastoreo y construcción de carreteras y obras hidráulicas sin medidas de protección de taludes. En la Fig. 1 se muestra la ubicación del reservorio San Lorenzo.
El reservorio San Lorenzo tiene una capacidad de almacenamiento total de 258 MMC y una capacidad de almacenamiento útil de 252,7 MMC; la superficie del espejo de agua es de $16 \mathrm{~km}^{2}$ y el volumen muerto inicial fue de 3,0 MMC. Actualmente, la sedimentación del reservorio San Lorenzo, durante los 50 años de operación, es del orden de los 51,52 MMC, representando el 20,4\% de su volumen útil.

Para evaluar el comportamiento de los sedimentos y la disminución de la capacidad de almacenamiento del embalse San Lorenzo, se ha desarrollado la tesis que lleva por título "Evaluación de la sedimentación del reservorio San Lorenzo y comportamiento de la capacidad de almacenamiento cumplidos los 50 años de su vida útil. "Su objetivo es conocer en el tiempo y espacio el comportamiento de la capacidad de almacenamiento de la represa San Lorenzo; como objetivos específicos el estudio quiere precisar el volumen total de sedimento del reservorio, desde su puesta en funcionamiento a la fecha (1959 - 2008) y determinar la capacidad del volumen útil actual.

\section{Materiales y métodos}

\section{Información cartográfica}

- Carta nacional: Reservorio San Lorenzo Esc: 1/10,000

- Mapas de la cuenca Quiroz y Chipillico Esc: 1/200,000.

- Planos de planta del reservorio San Lorenzo Esc: $1 / 200,000$ y $1 / 400$.

- Plano de planta perimetral y ubicación de las secciones maestras.

\section{Información batimétrica}

- Informe de los planos de diseño, año 1959.

- Informe de la batimetría, año 1978.

- Informe de la batimetría, año 1998.

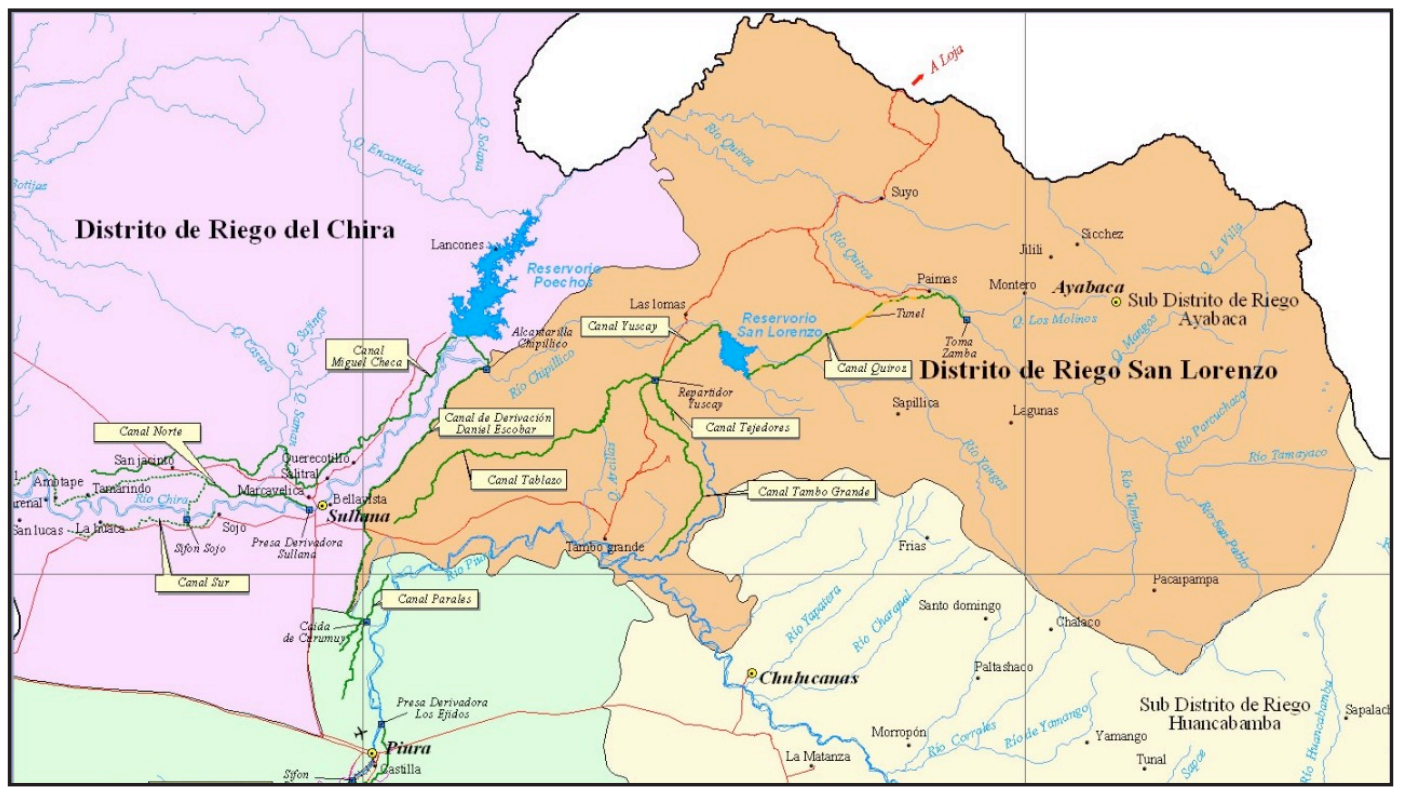

Figura 1. Mapa de ubicación del reservorio San Lorenzo 
- Informe de la batimetría, año 2004.

- Informe de la batimetría, año 2008.

\section{Información hidrometeoro lógica}

- Registros mensuales de caudales de los años 1954-2008 de los ríos Quiroz, Chipillico y Chira.

- Registros mensuales del transvase del río Quiroz al reservorio San Lorenzo.

\section{Equipo topográfico e hidrográfico}

- 01 Estación Total TOPCON modelo GPT 7000L.

- 03 bastones con sus respectivas primas.

- 01 ecosonda graficadora digital GPSMAP 188C SOUND.

\section{Material de escritorio y equipos misceláneos}

- 01 Computadora y software.

- Cámara fotográfica.

- GPS navegador.

- Otros.

\section{Metodología}

- Fase de campo.

- Reconocimiento del área de estudio.

- Trabajo de campo.

- Levantamiento topográfico.

- Levantamiento batimétrico.

- Muestreo de sedimento depositado en el lecho del río.

- Fase gabinete.

- Recopilar las informaciones de las batimetrías y procesamiento de datos.

\section{Resultados y discusión}

\section{Análisis hidrológico}

Para la determinación de la magnitud de las crecidas o caudales y sus proyecciones a futuro se aplicó la teoría de probabilidades con cierto grado de incertidumbre. Los métodos probabilísticos se basan en el estudio de los registros históricos de caudales, que dependerán, principalmente, del tamaño y la calidad de la información hidrológica (precipitación y/o descarga) que se haya registrado y la experiencia del profesional.

Resultado del análisis de consistencia.

Del análisis de los histogramas y de las curvas doble masa de las estaciones Ardilla y Paraje Grande se tiene las siguientes conclusiones:

Estación Ardilla. Considerándose como estación índice, se ha efectuado este análisis para verificar la consistencia de la serie histórica. Apreciándose que no existen variaciones bruscas de la pendiente de dicho gráfico, por lo que este análisis confirma la consistencia final de la información de caudal medio mensual en la cuenca del río Chira, es decir el análisis desarrollado ha sido adecuado.
Estación Paraje grande. El análisis de doble masa presenta variabilidad de pendientes, por lo que es necesario realizar la corrección de la información inconsistente.

Estación Lagartera. Al igual que la estación anterior, el análisis de doble masa presenta variabilidad de pendientes.

\section{Análisis de saltos}

El análisis estadístico de presencia de saltos de las estaciones consideradas en el estudio, da los siguientes resultados: En las estaciones Lagartera y Paraje Grande el salto en la media y la desviación estándar es significativa, por lo tanto se debe corregir la información de ambas estaciones.

\section{Corrección de la información inconsistente}

En base a los resultados del análisis de consistencia, se ha procedido a la corrección de información del periodo dudoso. En algunos casos, se ha considerado la eliminación de la información del periodo inconsistente.

\section{Conclusión y extensión de la información}

Una vez corregida o eliminada la información inconsistente, se ha procedido a la conclusión y/o extensión del registro histórico. Para tal fin se ha elegido como periodo de trabajo el que comienza en el año 1954 y termina en el año 1981 (27 años). Se han completado los datos mediante una correlación simple entre las estaciones Lagartera, Ardilla y Paraje Grande, utilizando el programa SIH (Sistema de Información Hidrológica) desarrollado por la intendencia de recursos hídricos del ministerio de agricultura de Perú.

\section{Pruebas de bondad de ajuste}

La prueba de bondad de ajuste consiste en verificar estadísticamente si la frecuencia empírica analizada se ajusta a una determinada función de probabilidades teóricas seleccionadas a priori, en base a los parámetros estimados de los valores muestrales.

Para averiguar cuán aproximada es la distribución empírica a la teórica, se ha optado por la prueba estadística de Smirnov-Kolmogorov con un nivel de significación del $95 \%$ aplicada a las distribuciones de frecuencias normal, utilizándose el software SIH. Los resultados son que los datos completados y extendidos de las estaciones Lagartera y Paraje Grande se ajustan a una Distribución Normal con un nivel de significancia del $5 \%$ o $95 \%$ de probabilidad, por lo tanto esta información se puede emplear para determinar los volúmenes de arrastre de sedimentos por los ríos Quiroz y Chipillico hacia el reservorio San Lorenzo.

\section{Resultado de la batimetría del reservorio San Lorenzo} Los resultados de la batimetría del reservorio San Lorenzo son que este tiene una profundidad máxima de $38,00 \mathrm{~m}$ (comprendido entre el nivel máximo 290,00 msnm y 
el nivel mínimo 252,00 msnm), ubicada en el área de emplazamiento de la estructura hidráulica de descarga; un volumen de regulación de 201.174.372,33 $\mathrm{m}^{3}$ y una superficie de espejo de agua de 15.459.029,12 $\mathrm{m}^{2}$.

Resultados de las batimetrías del reservorio San Lorenzo.

En la siguiente Tabla se ha resumido las batimetrías realizadas en los años de 1979, 1998, 2004 y 2008 (Tabla

Tabla 1. Volumen total obtenido por las batimetrías

\begin{tabular}{|l|l|}
\hline Batimetría & Volumen Total $\left(\mathbf{m}^{3}\right)$ \\
\hline Plano Diseño & $252.695 .000,0$ \\
\hline Batimetría 1978 & $217.332 .005,0$ \\
\hline Batimetría 1998 & $190.839 .745,5$ \\
\hline Batimetría 2004 & $199.073 .695,1$ \\
\hline Batimetría 2008 & $201.174 .372,3$ \\
\hline
\end{tabular}

\section{1 y Figura 2).}

La batimetría del año 2008 presenta mayor confiabilidad debido a que este trabajo se realizó con equipos actualizados, las cuales nos permiten una mayor precisión en la estimación del volumen total del reservorio San Lorenzo; hecha esta aclaración, los resultados obtenidos en dicha batimetría fueron utilizados para la corrección del método de Graf.

Para determinar las características hidráulicas del tramo evaluado e identificar la geometría compleja, en los ríos Quiroz y Chipillico, se utilizó el modelo HEC-RAS, desarrollado por Hydrologic Engineering Center-River Analysis System. En primer lugar, se estableció la sección de río en la zona de estudio, para lo cual se realizaron las mediciones del cauce y riberas del tramo evaluado en ambos ríos, con secciones transversales de 50 metros, sobre una longitud total de 200 metros. En segundo lugar, se estableció los caudales medios mensuales.

\section{Resultados de la aplicación del modelo hidráulico}

En el tramo evaluado, los ríos presentan las siguientes características hidráulicas:

El tirante mínimo se presenta en los meses de estiaje, con una velocidad mínima y, en algunos, los valores son cero debido a que el río llega a secarse completamente, como el caso del río Chipillico. En los meses de avenida se presentan las máximas. Las secciones transversales que observamos son, por lo general, muy irregulares. El área de la sección y la velocidad del flujo dependen del caudal presente para cada mes del año.

\section{Estimación del transporte de sedimento}

Para la determinación del transporte total de sedimentos de los ríos Quiroz y Chapillico se usó la metodología desarrollada por Graf.

\section{Validación al método de Graf}

La aplicación del método de Graf y de sus fórmulas de transporte de sedimentos en los cauces de los ríos Quiroz y Chipillico, consiste en definir una sección transversal hidráulica típica del río que pueda considerarse representativa del tramo de ingreso a su embalse o de los tributarios principales. Teniendo como base las características de los sedimentos dominantes en el tramo de interés, se determinan sus parámetros representativos a partir de la curva granulométrica correspondiente.

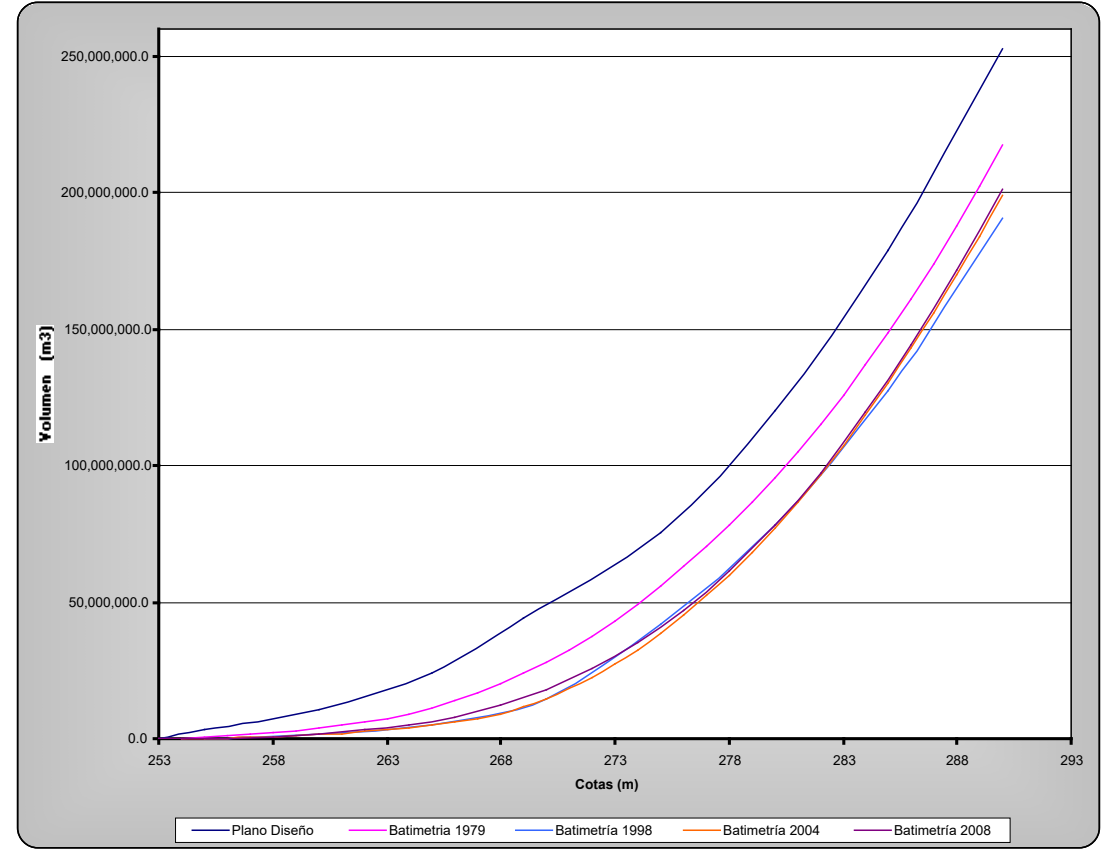

Figura 2. Pérdida del volumen total según las batimetrías. Determinación de las características hidráulicas de los ríos Quiroz y Chipillico 
En función de las características hidráulicas, morfológicas y de sedimentación del cauce, se procede a estimar la capacidad de transporte total del material del lecho. Su aplicación consigue satisfacer los siguientes requerimientos:

Permite el cálculo de la descarga de los sedimentos totales, con una precisión aceptable.

Calcula los sedimentos totales con los datos que se obtienen en una sola sección o en un tramo corto.

Aplicable a secciones de cauces irregulares.

Utiliza los parámetros hidráulicos medidos en la sección.

\section{Resultados por el método de Graf}

Los resultados de la aplicación del método de Graf, para la determinación del transporte total de sedimentos de los ríos Quiroz y Chapillico, los encontramos en la Tabla 2. El volumen de sedimento transportado por el canal Quiroz fue determinado proporcionalmente en función al caudal, debido a que los sedimentos de arrastre están directamente relacionados con este.

Si bien es cierto que el volumen de sedimento total es el valor máximo que podría transportar el cauce, siempre y cuando exista la disponibilidad de sedimentos en las

Tabla 2. Volúmenes obtenidos por método de Graf

\begin{tabular}{|l|l|}
\hline Río & $\begin{array}{l}\text { Volumen transportado } \\
\text { (MMC) (Método de Graf) }\end{array}$ \\
\hline Río Quiroz & $4.263,24$ \\
\hline Río Chipillico & $4.905,76$ \\
\hline Canal Quiroz & $2.021,96$ \\
\hline
\end{tabular}

subcuencas de los ríos Quiroz y Chipillico. El método de Graf menciona que los volúmenes obtenidos no son los que el río transporta, sino es la capacidad máxima que pueda trasportar un río. Hecha esta aclaración, los valores obtenidos por el método de Graf fueron revisados por un factor de corrección, obtenidos por la división entre los valores del volumen total de la batimetría del último año (2008) y los valores de los volúmenes transportados por el río Chipillico y el canal Quiroz.

Los resultados de aplicar el factor de corrección a los volúmenes obtenidos por el método de Graf a los ríos Quiroz y Chipillico y al canal Quiroz, se presentan en las Tablas 3 y 4.
Tabla 3. Volúmenes corregidos

\begin{tabular}{|l|l|}
\hline Río & $\begin{array}{l}\text { Volumen transportado } \\
\text { (MMC) (Método de Graf) }\end{array}$ \\
\hline Río Quiroz & $4.263,24$ \\
\hline Río Chipillico & $4.905,76$ \\
\hline Canal Quiroz & $2.021,96$ \\
\hline
\end{tabular}

Tabla 4. Volúmenes de sedimento aportado por el río Chipillico y el canal Quiroz hacia el reservorio San Lorenzo

\begin{tabular}{|l|l|}
\hline Río & $\begin{array}{l}\text { Volumen transportado } \\
\text { (MMC) (Método de Graf) }\end{array}$ \\
\hline Río Chipillico & $36.483 .535,39$ \\
\hline Canal Quiroz & $15.037 .092,29$ \\
\hline Total & $51.520 .627,67$ \\
\hline
\end{tabular}

En la Tabla 3 se observa que el volumen de sedimentos transportados por el río Chipillico es mayor que el del río Quiroz; esto es debido a que la subcuenca es muy sensible a pequeños cambios climáticos. En los meses de estiaje, la subcuenca del río Chipillico presenta caudales mínimos y, en algunos meses, llega a secarse completamente. En los meses de avenida los caudales se incrementan notoriamente. Estos incrementos bruscos en el caudal hacen que los sedimentos de arrastre se incrementen debido a la inestabilidad del cauce.

\section{Pérdida del volumen total del reservorio San Lorenzo}

En las Tablas 5 y 6 se muestra la comparación de la pérdida de volumen total en $\mathrm{m}^{3}$ y en porcentaje en el embalse San Lorenzo, según las batimetrías realizadas.

En la Fig. 3, se muestra la curva de ajuste para la pérdida de la capacidad del volumen total en el embalse San Lorenzo. La reducción de la capacidad del volumen total del reservorio, se ajusta a una curva polinómica de tercer orden cuya ecuación es (Fig. 4):

$y=0,0002 x^{3}-0,032 x^{2}-0,0255 x+251,19$

$\mathrm{Y}$ el valor de $r^{2}$ es:

$r^{2}=0,9906$

Los sedimentos acumulados en el reservorio San Lorenzo se ajustan a una curva polinomial de tercer orden cuya ecuación es:

$y=0,0002 x^{3}-0,032 x^{2}-0,0255 x+251,19$

$\mathrm{Y}$ el valor de $r^{2}$ es:

$r^{2}=0,9906$

Fc $=\frac{\text { vol. } \text { total de la batimetria del } 2008}{\text { vol. transp. por el río Chipillico y el canal Quiroz }}$

Factor de corrección $=0,00744$ 
Tabla 5. Comparación de la pérdida de volumen total en $\mathrm{m}^{3}$

\begin{tabular}{|c|c|c|c|c|}
\hline Años & $\begin{array}{c}\text { Volumen de } \\
\text { Reservorio } \\
\text { Según las } \\
\text { Batimetrías } \\
\left.\mathbf{( m}^{\mathbf{3}}\right)\end{array}$ & $\begin{array}{c}\text { Volumen de } \\
\text { Reservorio } \\
\text { Según Método } \\
\mathbf{d e ~ G r a f ~} \\
\mathbf{( m}^{\mathbf{3}} \mathbf{n}\end{array}$ & $\begin{array}{c}\text { Pérdida Vol. Reser. con } \\
\text { respecto al Vol. Inicial } \\
\text { (Según Batimetrías) } \\
\mathbf{( m}^{\mathbf{3}} \mathbf{)}\end{array}$ & $\begin{array}{c}\text { Pérdida Vol. Reser. con } \\
\text { respecto al Vol. Inicial } \\
\text { (Según Método Graf) } \\
\mathbf{( m}^{\mathbf{3}} \mathbf{)}\end{array}$ \\
\hline $\begin{array}{c}\text { Diseño (1985) } \\
1978\end{array}$ & $\begin{array}{c}252.695 .000,0 \\
217.332 .005,0\end{array}$ & $240.530 .007,6$ & $35.262 .995,0$ & $12.164 .992,4$ \\
\hline 1998 & $190.839 .745,5$ & $213.658 .154,0$ & $61.855 .254,5$ & $39.036 .846,0$ \\
\hline 2004 & $199.073 .695,1$ & $205.613 .932,9$ & $53.621 .304,9$ & $47.081 .067,1$ \\
\hline 2008 & $201.174 .372,3$ & $201.174 .372,3$ & $51.520 .627,7$ & $51.520627,7$ \\
\hline
\end{tabular}

Tabla 6. Comparación de la pérdida del volumen en \%

\begin{tabular}{|c|c|c|c|c|}
\hline Años & $\begin{array}{l}\text { Volumen de } \\
\text { Reservorio } \\
\text { Según las } \\
\text { Batimetrías } \\
\quad\left(\mathrm{m}^{3}\right)\end{array}$ & $\begin{array}{c}\text { Volumen de } \\
\text { Reservorio } \\
\text { Según Método } \\
\text { de Graf } \\
\left(\mathbf{m}^{3}\right)\end{array}$ & $\begin{array}{c}\text { Pérdida Vol. Reser. } \\
\text { Con respecto al } \\
\text { Vol. Inicial (Según } \\
\text { Batimetrías) } \\
(\%)\end{array}$ & $\begin{array}{l}\text { Pérdida Vol. Reser. } \\
\text { Con respecto al Vol. } \\
\text { Inicial (Según Método } \\
\text { Graf) } \\
(\%)\end{array}$ \\
\hline $\begin{array}{c}\text { Diseño (1985) } \\
1978\end{array}$ & $\begin{array}{l}252.695 .000,0 \\
217.332 .005,0\end{array}$ & $240.530 .007,6$ & 14,0 & 4,8 \\
\hline $\begin{array}{l}1998 \\
2004\end{array}$ & $\begin{array}{l}190.839 .745,5 \\
199.073 .695,1\end{array}$ & $\begin{array}{l}213.658 .154,0 \\
205.613 .932,9\end{array}$ & $\begin{array}{l}24,5 \\
21,2\end{array}$ & $\begin{array}{l}15,4 \\
18,6\end{array}$ \\
\hline 2008 & $201.174 .372,3$ & $201.174 .372,3$ & 20,4 & 20,4 \\
\hline
\end{tabular}

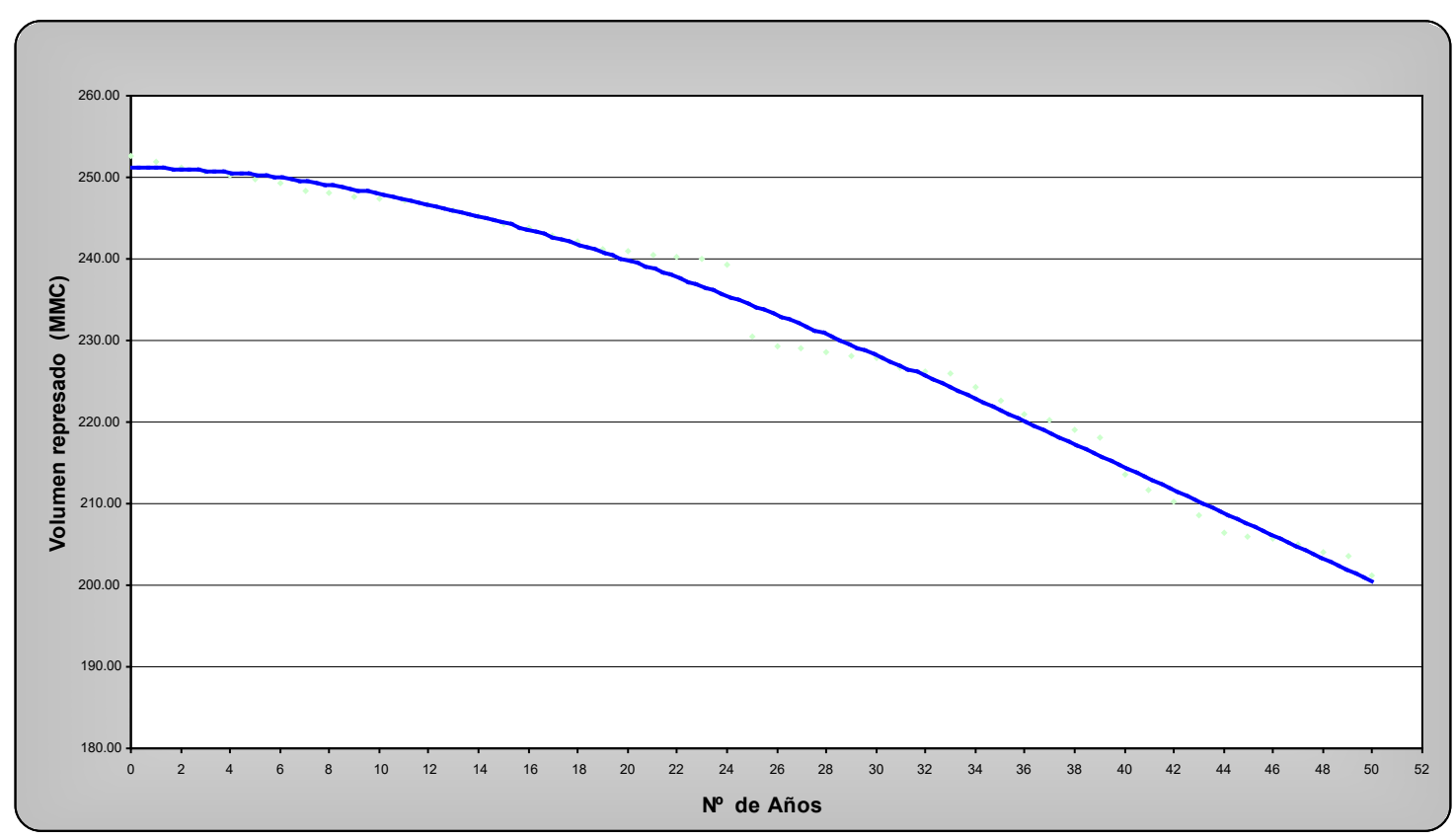

Figura 3. Curva de ajuste para la pérdida de capacidad de volumen del reservorio San Lorenzo.

La pérdida del volumen disponible en el reservorio San Lorenzo es un proceso progresivo debido a la ocupación de los sedimentos transportados por los ríos Quiroz y Chipillico (Fig. 5). A la fecha el reservorio San Lorenzo presenta una sedimentación del $20,4 \%$ de su volumen útil, es decir 51,52 MMC, lo que reduce su capacidad de almacenamiento y limita la racionalización del uso del agua, sobre todo en la segunda campaña agrícola. 


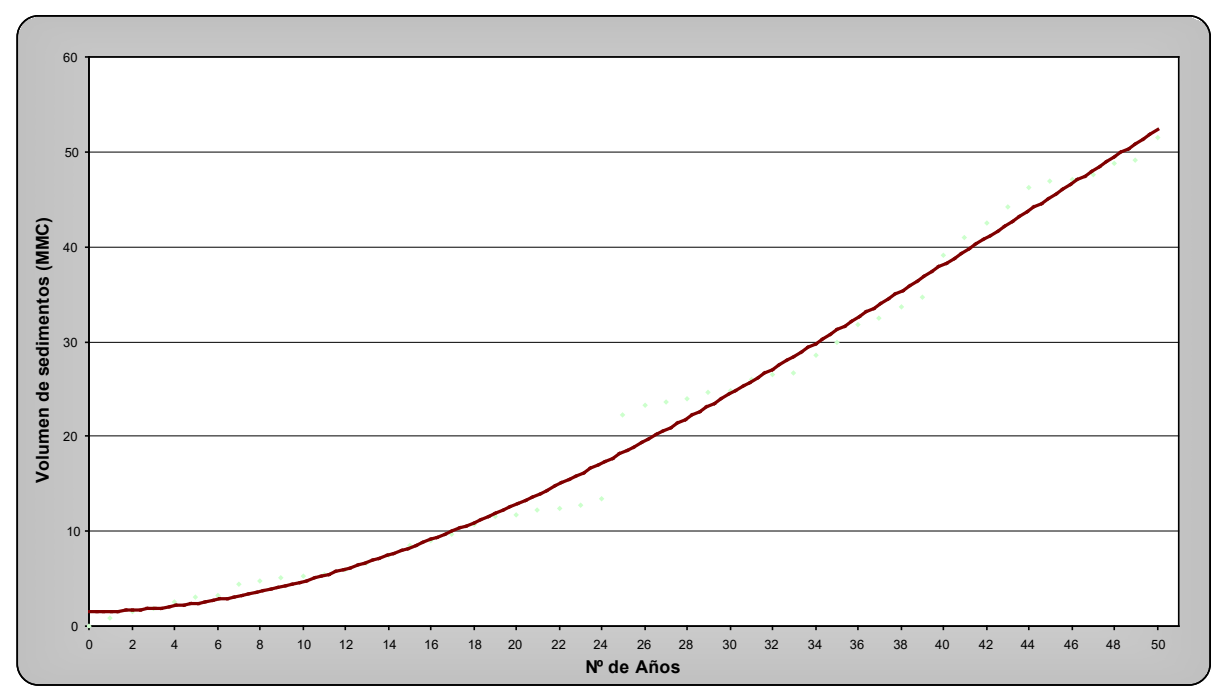

Figura 4. Curva de ajuste para la acumulación de sedimentos - reservorio San Lorenzo

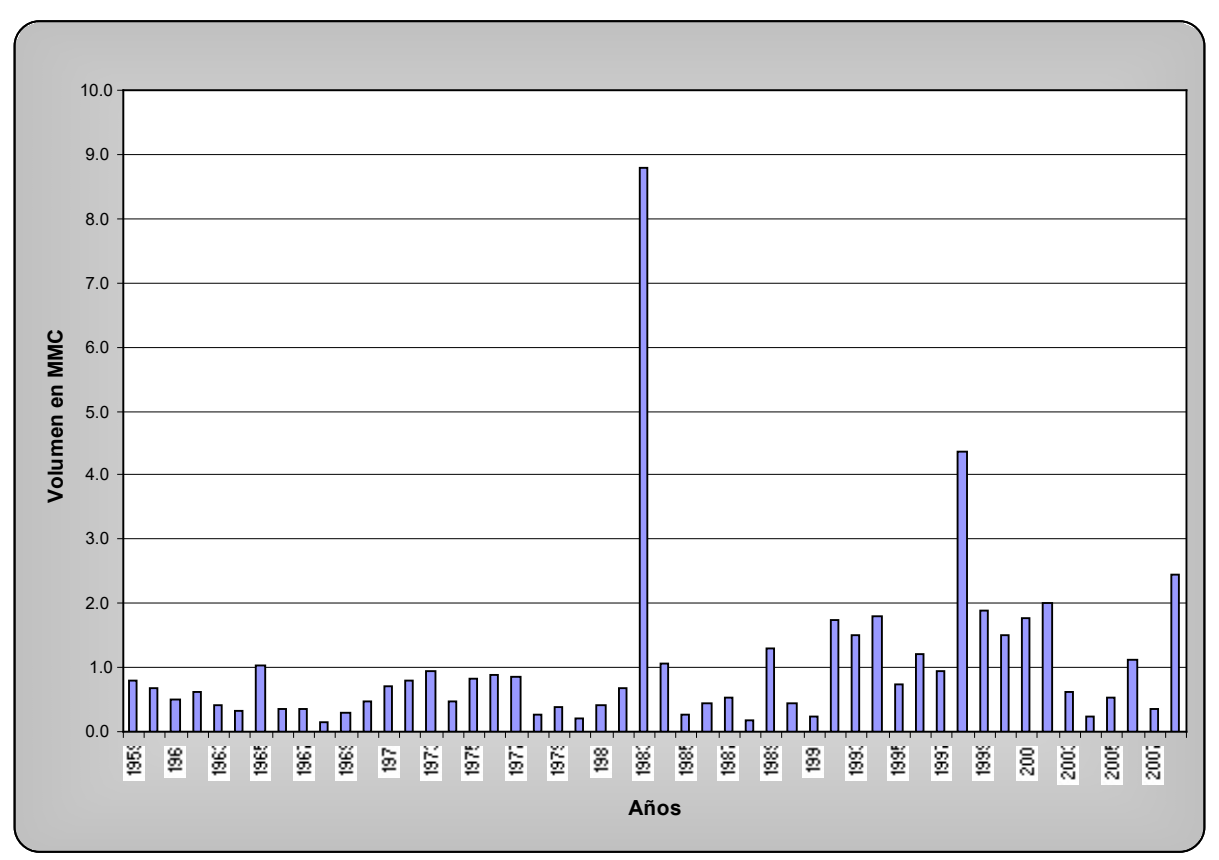

Figura 5. Volumen anual de sedimentos acumulados en la represa San Lorenzo

\section{Conclusiones}

La pérdida del volumen del reservorio San Lorenzo para un año normal es de $1,55 \%$ del volumen útil, esto quiere decir que cada año en promedio se está perdiendo $789.569,45 \mathrm{~m}^{3}(0,78 \mathrm{MMC})$ del volumen total. Y en los años de ocurrencia del fenómeno de El Niño la pérdida del volumen esta entre 5 a $15 \%$. Transcurridos y cumplidos los 50 años de vida útil, podemos decir que el reservorio San Lorenzo ha cumplido las expectativas de diseño. Quedando así un 79,6 \% de su volumen para las próximas generaciones.

\section{5 . Literatura citada}

Lezcano-Rocha. 1969. Transporte de Sedimentos. LimaPerú: Publicación UNI. 